\title{
Piégeage des Mouches des Fruits (Diptera : Tephritidae) À Base D'extraits de Ocimum Basilicum L. (Lamiaceae) : Cas de Bactrocera Dorsalis, Principal Ravageur de Mangues en Côte d'Ivoire
}

\author{
N'Dépo O. R., \\ Tano D. K. C., \\ Laboratoire d'Amélioration de la Protection Agricole, \\ Université Jean Lorougnon Guédé, Dalao, Côte-d'Ivoire \\ Hala N., \\ Adopo N. A.,
}

Centre National de Recherche Agronomique, Abidjan, Côte d'Ivoire

Kouassi K. P.,

Laboratoire de Zoologie et Biologie Animale, UFR Biosciences, Université Felix Houphouët Boigny,Abidjan Côte d'Ivoire

Doi:10.19044/esj.2020.v16n3p458 ～URL:http://dx.doi.org/10.19044/esj.2020.v16n3p458

\section{Résumé}

Les cultures fruitières, en particulier la mangue en Côte d'Ivoire, sont sous la menace des mouches des fruits. Les dégâts se sont accrus avec l'invasion de Bactrocera dorsalis. En outre, la baisse des pertes en vergers de manguiers exige l'utilisation du méthyl eugénol pour sa détection et le suivi $\mathrm{du}$ monitoring de sa population. Malheureusement, les attractifs sexuels spécifiques de mouches sont inaccessibles aux producteurs de la Côte d'Ivoire. L'objectif de cette étude est d'évaluer l'efficacité de Ocimum basilicum dans de la capture de $B$. dorsalis. Ces trois formulations du basilic (feuilles fraîches malaxées, poudre de basilic et macérât du basilic) et le méthyl eugénol sont utilisées en piégeage dans les vergers de manguiers à Korhogo. Les pièges ont été relevés chaque semaine et chaque jour respectivement pour le Méthyl eugénol et les formulations du basilic. Les formulations ont permis de capturer une importante population de B. dorsalis $(99,41 \%)$ avec une prévalence (FTD) moyenne journalière de 10,141 individus/jour/piège et d'autres espèces de mouches de fruits. La poudre de basilic montre une rémanence plus longue ( $28,67 \pm 18,17$ jours) tandis que le macérât de basilic montre une capture hebdomadaire plus élevée (513,4 $\pm 72,34$ individus). Les captures moyennes les plus élevées ont été observées avec le Macérât de basilic (4419 \pm 1090 individus) et le Méthyl eugénol (4899,67 \pm 1511,74 individus). Le macérât de 
feuilles fraîches de basilic et la poudre de feuilles sèches de basilic peuvent être recommandées auprès des producteurs de mangues dans la lutte contre $B$. dorsalis.

Mots clés : Basilic, Méthyl eugénol, Formulations, Bactrocera dorsalis

\title{
Trapping of Fruit Flies (Diptera: Tephritidae) Based on Extracts of Ocimum Basilicum L. (Lamiaceae): Case of Bactrocera Dorsalis Main Pest of Mangoes
}

\author{
N’Dépo O. R., \\ Tano D. K. C.,
}

Laboratoire d'Amélioration de la Protection Agricole,

Université Jean Lorougnon Guédé, Dalao, Côte-d'Ivoire

Hala $N$.,

Adopo N. A.,

Centre National de Recherche Agronomique, Abidjan, Côte d'Ivoire

Kouassi K. P.,

Laboratoire de Zoologie et Biologie Animale, UFR Biosciences,

Université Felix Houphouët Boigny,Abidjan Côte d'Ivoire

Abstract

Fruit crops, especially mangoes in Côte d'Ivoire are threatened by the fruit flies. The damage has increased with the invasion of Bactrocera dorsalis. In addition, the decline in mango orchard losses requires the use of methyl eugenol for the detection and monitoring of Bactrocera population. Unfortunately, the sex-specific attractants of flies are inaccessible to producers in Côte d'Ivoire. The aims of this study is to evaluate the efficacy of Ocimum basilicum in the capture of $B$. dorsalis. Three basil formulations (fresh mixed leaves, basil powder and basil macerate) and methyl eugenol are used for trapping in mango orchards in Korhogo. The captured insects were collected weekly and daily for methyl eugenol and basil formulations respectively. The formulations captured a large population of $B$. dorsalis $(99.41 \%)$ with an average daily prevalence (FTD) of 10,141 individuals / day / trap and other fruit fly species. Basil powder has the longest persistence ( $28.67 \pm 18.17$ days) and basil macerate has the highest weekly catch (513.4 \pm 72.34 individuals). The highest average catches were observed with basil macerate $(4419 \pm 1090$ 
individuals) and methyl eugenol (4899.67 \pm 1511.74 individuals). Fresh basil leaf macerate and dry basil leaf powder may be recommended to mango growers in the control of $B$. dorsalis.

Keywords : Basil, Méthyl eugénol, Formulations, Bactrocera dorsalis

\section{Introduction}

Les mouches de fruits (Tephritidae) constituent un problème majeur dans la production des fruits dans le monde et particulièrement en Afrique de l'ouest (Vayssières et al., 2005). Ces espèces sont très préoccupantes par les dégâts directs qu'elles provoquent sur les fruits et légumes lors de l'alimentation de leurs larves et la perte d'opportunité de marchés d'exports à travers la restriction due à la quarantaine imposée par les pays importateurs (Ekesi et al., 2007 ; N’Dépo et al., 2009). Parmi les 500 genres que renferme la famille des Tephritidae, le genre Bactrocera est considéré comme le plus dévastateur de fruits et légumes dans le monde (White and Elson-Harris, 1992 ; Ekisi et al., 2006). C'est le cas de Bactrocera dorsalis, originaire de Sri Lanka en Asie qui a été introduite accidentellement en Afrique selon Lux et al. (2003) (cité dans Drew et al., 2005). Cette espèce invasive a colonisé plus de 22 pays africains, où elle est devenue un ravageur économiquement nuisible (De Meyer et al., 2007). Elle représente une réelle menace pour la filière mangue en Afrique de l'Ouest, particulièrement en Côte d'Ivoire. Face à cette menace, les producteurs et exportateurs sont désemparés sans moyen de lutte rapide et tentent de délaisser les vergers au profit d'autres cultures (N'Dépo, 2010). Aujourd'hui, Bactrocera dorsalis a été identifiée en Europe, plus précisément en Italie (Francesco et al., 2018). Elle attaque plus de 20 espèces de fruits parmi lesquels la mangue apparait la plus attaquée (Ivan et al., 2008 ; N’Dépo et al., 2010). Elle a été recensée en 2005 en Côte-d'Ivoire (Hala et al., 2006 ; N'Dépo, 2006) et est responsable de plus de $80 \%$ de dégâts des mangues (N’Dépo et al., 2009). Au Cameroun, des travaux menés en vergers de manguiers dans la localité de Malang, ont révélé qu'elle est majoritaire $(66,55 \%)$ dans les pièges installés et dans la mangue $(98,5 \%)$ (Tinkeu et al., 2010). Au Togo, Amevoin et al. (2009) ont constaté que B. dorsalis présente une prévalence moyenne (23,68 individus/ piège/ Jour) supérieure à celle des autres espèces capturées et sa période de pullulation coïncide avec celle de la maturation des mangues sa principale plante-hôte. Dans la localité de Daloa au Centre-Ouest de la côte d'Ivoire, zone de faibles productions fruitières, des études récentes sur les mouches des fruits, ont révélé une forte population de B. dorsalis comparativement aux autres espèces de mouches (Achiépo, 2019 ; Dramane, 2019). Cette mouche est fortement attirée par le Méthyl eugénol (attractif sexuel) qui est indispensable à sa détection et au monitoring de sa population (N'Dépo et al., 2015 ; Coulibaly, 2015). Malheureusement, les 
attractifs sexuels des mouches de la mangue sont inaccessibles aux producteurs, en particulier ceux en Côte d'Ivoire. L'objectif de cette étude est d'utiliser un appât local attirant efficacement B. dorsalis et capable de lutter à long terme contre ce déprédateur.

\section{Matériel et Méthodes}

\subsection{Site d'étude}

Les essais ont été conduits pendant 06 mois (de Mars à Août 2017, en zone de production de mangue, plus précisément à la station de Lataha ( $9^{\circ} 34^{\prime}$ de longitude Nord ; $5^{\circ} 37^{\prime}$ de latitude Ouest) à Korhogo. Cette station spécialisée dans la production fruitière, regorge des vergers d'anacardiers, de papayers, d'agrumes et de manguiers de même que le karité, le néré et autres arbres fruitiers. La température moyenne annuelle de la station est de 24,42 \pm $0,5^{\circ} \mathrm{C}$ alors que la pluviométrie est de $928,85 \mathrm{~mm} / \mathrm{an}$.

\subsection{Méthodes}

\subsubsection{Dispositif Expérimental}

2.2.1.1 Etude de la durée d'efficacité des différentes formulations du basilic

Trois formulations de feuilles et le méthyl eugénol (témoin) ont été utilisées comme attractifs dans les pièges. Trois pièges par formulation, ont ensuite été installés dans trois vergers de manguiers avec une distance minimale de $50 \mathrm{~m}$ entre les deux formulations suivant la méthode standard (Vayssières et al., 2009 ; Ouedraogo et al., 2011 ; Daniel et al., 2015) et une distance minimale de $500 \mathrm{~m}$ entre les vergers. Au total, 12 pièges ont été installés en vergers de manguiers. La prévalence des mouches (FTD), la capture moyenne de mouches par formulation et la rémanence des formulations ont été déterminées. Une rotation a été appliquée aux pièges toutes les semaines. Le macérât a été complété à $250 \mathrm{ml}$ tous les 2 jours du fait du caractère volatil de l'éthanol. Le piégeage a été effectué sur une période de 6 mois (de Mars à Août 2017) en verger de manguiers pendant la maturation des mangues.

\subsubsection{Piégeage de mouches aux attractif formulés et au méthyl eugénol}

Un test d'efficacité et de la durée d'attraction a été réalisé avec trois formulations du basilic [Feuille fraîche malaxée, la Poudre de basilic et le macéré éthanolique du basilic (Macérât de basilic)] comparé à un témoin (méthyl eugénol) au cours du piégeage de B. dorsalis. Le test d'efficacité a consisté à l'attraction et à la capture de $\mathrm{B}$. dorsalis par les formulations au cours de l'expérience. La rémanence a été évaluée sur la base de la durée moyenne d'efficacité (durée de capture de mouches) des formulations. 


\subsubsection{3- Attractifs formulées aux feuilles de basilic}

\section{a)- Formulation "Feuilles fraîches malaxées" du basilic (FFM)}

Des feuilles du basilic ont été prélevées sur la plante et pesées au moyen d'une balance électronique de marque OHAUS. Vingt (20) grammes de feuilles ont été malaxés ou écrasés à l'aide d'un mortier et d'un petit pilon en porcelaine. La "pâte " obtenue a été insérée dans la nacelle située sous le couvercle du piège à sec (Tephri Trap) pour attirer les mouches. Ces dernières en y pénétrant ont été tuées par le Dichlorvos (DDVP), insecticide déposé au fond du piège. Les pièges ont été relevés chaque jour. L'attractif FFM a été renouvelé lorsqu'il n'y avait plus de capture dans le piège alors que le Dichlorvos est renouvelé chaque mois. La durée de capture et le nombre d'individus ont été renseignés sur une fiche de suivi.

\section{b)- Formulation "Poudre de basilic" (PB)}

Quelques feuilles ont été récoltées et pesées. Vingt (20) grammes de matériel végétal ont été également prélevés, puis placés dans une étuve à une température de $50{ }^{\circ} \mathrm{C}$ pendant 72 heures pour le séchage. Les feuilles sèches ont été ensuite broyés à l'aide d'un broyeur électrique de marque BOSCH AXT 25 TC. La poudre a été récupérée et répartie en deux poignées de 10 grammes. Chaque poignée de poudre a été mise dans un pli confectionné à partir du papier filtre [papier Whatman (3MM)] avec des perforations pour faciliter la diffusion des composants du basilic. Les plis et le Dichlorvos ont été ensuite déposés au fond du piège à sec. Les pièges ont été relevés chaque jour. Cet appât a été renouvelé lorsqu'il n'y avait plus de capture de mouches dans le piège (Tephri-Trap). La durée de capture et le nombre d'individus ont été renseignés sur une fiche de suivi.

\section{c)- Formulation "Macérât de basilic" (MB)}

Vingt (20) grammes de feuilles ont été introduits dans un flacon de 1 $500 \mathrm{ml}$ auxquels ont été ajoutés $1000 \mathrm{ml}$ d'éthanol à $90^{\circ}$. La solution a été laissée au repos pendant 24 heures. Le macérât (solution verdâtre du basilic après macération) obtenu a été recueilli dans un deuxième flacon. Une quantité de $250 \mathrm{ml}$ (capacité du piège) de cette solution a été prélevée et mise dans le piège à appât liquide (Dome Trap). Les insectes qui y entrent, tombent et meurent par noyade dans le liquide. Les pièges ont été relevés chaque jour. La durée de capture et le nombre d'individus ont été renseignés sur une fiche de suivi.

\subsubsection{Attractif de référence (méthyl eugénol)}

Le méthyl eugénol est une formulation commerciale spécifique du genre Bactrocera. Cet attractif, lors du piégeage des mouches, est ôté de son emballage et inséré dans la nacelle du piège (Tephri-Trap). Le piégeage est 
réalisé en se basant sur la méthode de Ouattara (1998). Le piège est relevé toutes les semaines.

\subsubsection{Détermination de la prévalence des différentes espèces de mouches}

Les informations portant sur les effectifs des différentes espèces de mouches des fruits capturées dans les vergers de manguiers ont permis d'estimer leur niveau de prévalence en calculant le nombre moyen de mouches dans un piège en une journée (FTD) par la formulation suivante (Amevoin et al., 2009 ; Convention Internationale pour la Protection des végétaux [CIPV], 2016) :

CIPV], 2016) :

$$
\text { FTD }=\frac{F}{\mathrm{~T} \times \mathrm{D}} \quad \begin{aligned}
& \mathrm{F}=\text { nombre total de mouches capturées /espèce } \\
& \mathrm{T}=\text { nombre de pièges inspectés } \\
& \mathrm{D}=\text { nombre de jours d'exposition des pièges sur le terrain }
\end{aligned}
$$

\subsubsection{Exploitation statistique des données}

Les captures moyennes et hebdomadaires de Bactrocera dorsalis et la rémanence moyenne des différentes formulations ont été soumises à une analyse de variance (ANOVA) au moyen du logiciel STATISTICA v.7.1 au seuil de $5 \%$. Les moyennes obtenues ont été classées suivant le test de Tukey compte tenu de la taille inégale des échantillons.

\section{Résultats et discussion}

\subsection{Résultats}

3.1.1 Efficacité des formulations du basilic et du méthyl eugénol et la prévalence des Tephritidae dans les vergers de manguiers

Dans l'ensemble, les différentes formulations du basilic et du méthyl eugénol ont permis de capturer 9 espèces. Il s'agit de Dacus bivittatus, Trirhithrum coffeae, Dacus punctatifrons, Dacus langi, Ceratitis cosyra, Bactrocera dorsalis, Ceratitis punctata, Ceratitis bremii et Zeugodacus cucurbitae. B. dorsalis a été l'espèce majoritaire avec 21905 individus capturés sur un effectif total de 22034 mouches, soit $(99,41 \%)$. Les prévalences (FTD) moyennes journalières sont de 10,141 mouches/piège/jour pour $B$. dorsalis et de 0,002 à 0,124 mouches/piège/jour pour les autres espèces (Tableau 1). La zone de Korhogo est donc très infestée par les populations de $B$. dorsalis. L'analyse de variance a montré une différence hautement significative entre l'abondance de $B$. dorsalis et les autres espèces $(\mathrm{P}<0,01)$. La FFM a permis de capturer 2296 individus représentés uniquement par l'espèce $B$. dorsalis, soit $100 \%$. La PB a capturé 2588 mouches réparties en six (6) espèces. $B$. dorsalis, l'espèce majoritaire est estimée à 94,4 \% et les autres espèces composées de $D$. bivittatus, $D$. punctaifrons, $C$. punctata, $T$. coffeae, et $C$. cosyra, représentent $05,6 \%$ des 
individus capturés. Le MB a permis de capturer quatre (4) espèces dont $B$. dorsalis représente 99,91\% et les espèces, C. bremii, C. punctata, T. coffeae occupent une proportion de 0,09\%. En l'occurrence, l'attractif de référence, le méthyl eugénol a permis de capturé 6 espèces représentés par $B$. dorsalis avec 14699 individus, soit 99,93\%. Les autres espèces notamment $C$. cosyra, C. bremii, $C$. punctata et Z. cucurbitae et $T$. coffeae sont représentées par 10 individus, soit $0,07 \%$ de l'effectif total. Cette capture est très élevée avec le MB (4419 \pm 1090, 84 individus) et le méthyl eugénol (4899,67 \pm 1511,74 individus) comparativement à celle de la FFM et la PB (Figure 1). L'analyse de variance révèle une différence significative $(\mathrm{P} \leq 0,05)$ entre la capture moyenne des formulations.

Concernant la capture hebdomadaire moyenne des mouches par formulation, la même remarque a été observée. Cette capture hebdomadaire est plus faible avec la FFM et la PB. Par contre, elle est plus élevée avec le méthyl eugénol et le $\mathrm{MB}$ avec 415,88 \pm 125,96 mouches et 513,4 \pm 72,34 mouches respectivement (Figure 2). L'analyse de variance révèle une différence significative $(\mathrm{P} \leq 0,05)$ entre la capture hebdomadaire moyenne des formulations.

Tableau 1 : Espèces de mouches de fruits capturées par les différentes formulations à la station Lataha de Mars 2017 à Août 2017 et estimation de leur prévalence

\begin{tabular}{|c|c|c|c|c|c|}
\hline Espèces & $\begin{array}{c}\text { Type } \\
\text { d'attractifs } \\
\text { utilisés }\end{array}$ & $\begin{array}{l}\text { Nbre total } \\
\text { de jours } \\
\text { de capture }\end{array}$ & $\begin{array}{l}\text { Nbre total } \\
\text { de pièges } \\
\text { inspectés }\end{array}$ & $\begin{array}{c}\text { Nbre } \\
\text { d'individus } \\
\text { capturés }(\%)\end{array}$ & $\begin{array}{c}\text { Prévalence } \\
\text { (FTD) } \\
\text { (indv/jr/pi } \\
\text { ège) }\end{array}$ \\
\hline B. dorsalis, & $\begin{array}{l}\text { ME, MB, FM, } \\
\text { PB }\end{array}$ & 180 & 12 & $21905(99,41)$ & 10,141 \\
\hline T. coffeae & ME, MB, PB & 180 & 9 & $18(0,082)$ & 0,011 \\
\hline D. punctatifrons & $\mathrm{PB}$ & 180 & 3 & $02(0,009)$ & 0,004 \\
\hline D. langi & $\mathrm{PB}$ & 180 & 3 & $02(0,009)$ & 0,004 \\
\hline D. bivittatus & PB & 180 & 3 & $67(0,304)$ & 0,124 \\
\hline C. cosyra & PB & 180 & 3 & $03(0,014)$ & 0,006 \\
\hline C. punctata, & $\mathrm{ME}, \mathrm{MB}, \mathrm{PB}$ & 180 & 9 & $05(0,023)$ & 0,003 \\
\hline C. bremii & ME, MB & 180 & 6 & $32(0,145)$ & 0,030 \\
\hline Z. cucurbitae & ME & 180 & 3 & $01(0,005)$ & 0,002 \\
\hline
\end{tabular}




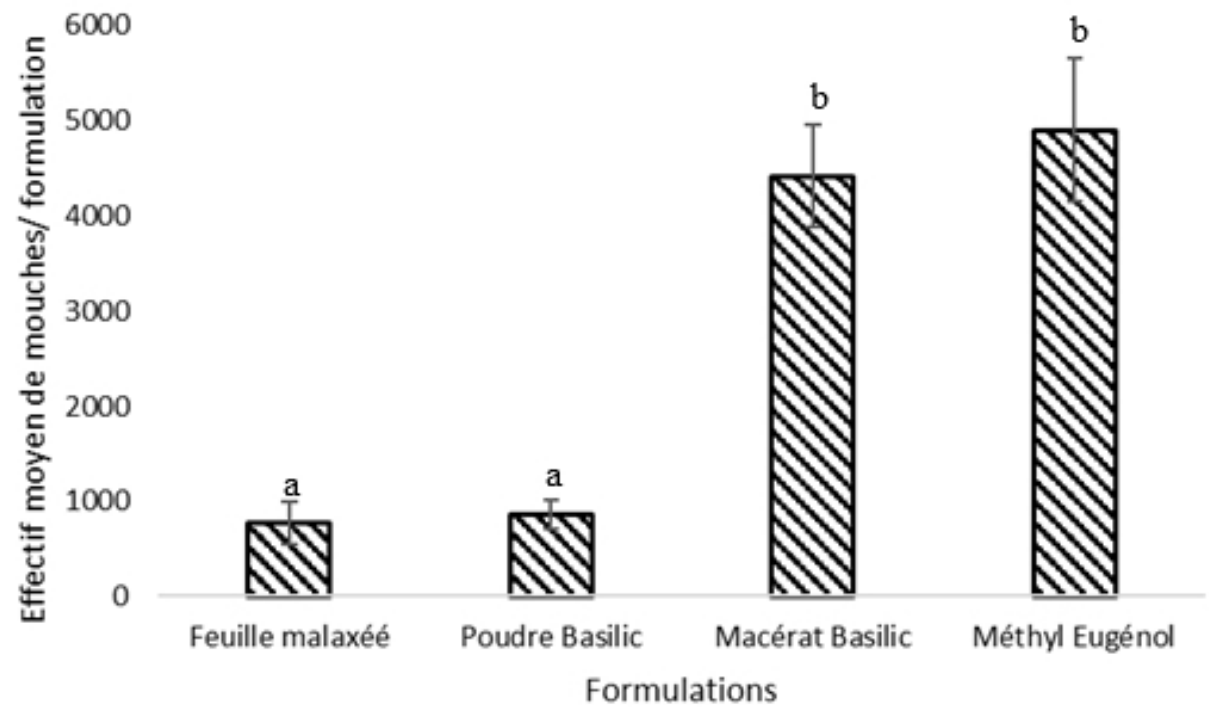

Figure 1 : Capture moyenne de Bactrocera dorsalis selon les formulations $\begin{array}{llll}\text { ANOVA à } 5 \% & \mathrm{dl}=3 & \mathrm{~F}=3,24 & 0,02\end{array}$

Les moyennes affectées de la même lettre ne sont statistiquement significatives

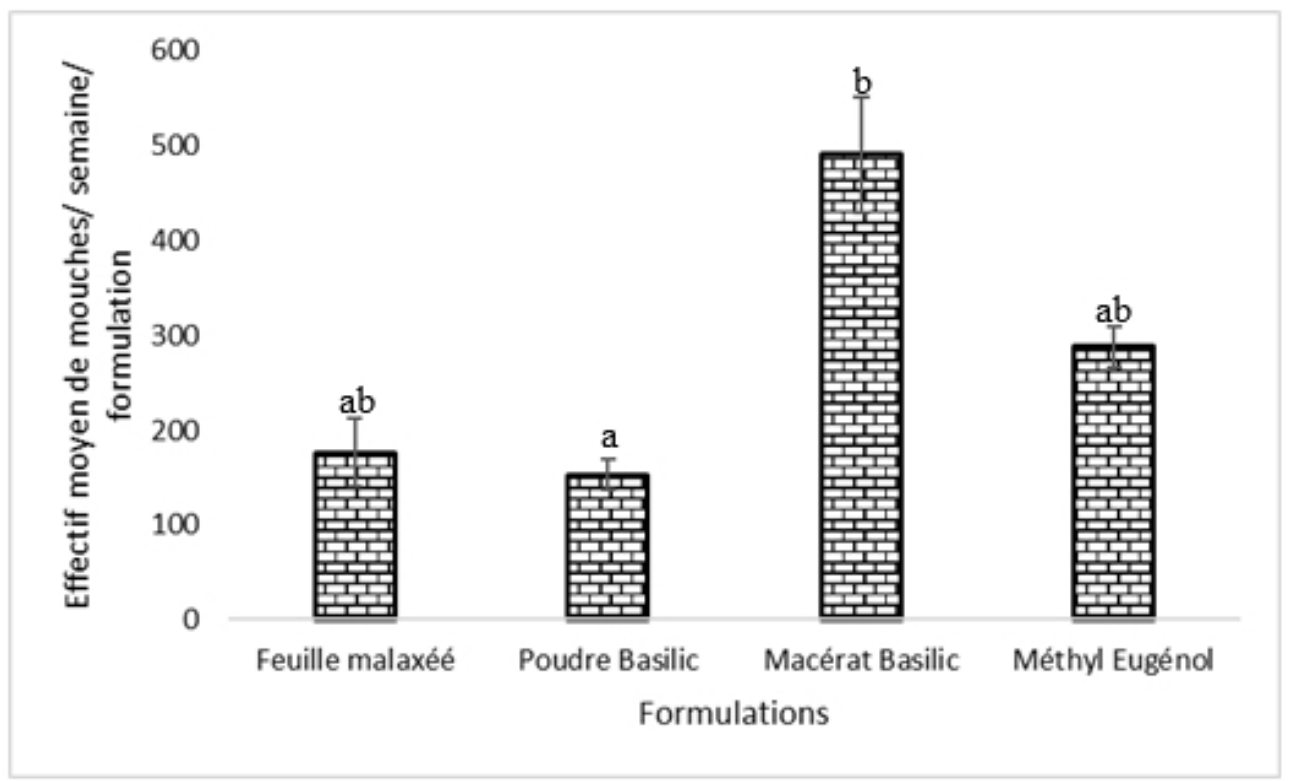

Figure 2 : Capture hebdomadaire moyenne de Bactrocera dorsalis selon les formulations ANOVA à $5 \% \quad \mathrm{dl}=3 \quad \mathrm{~F}=3,24 \quad \mathrm{P}=0,02$

Les moyennes affectées de la même lettre ne sont statistiquement significatives

\subsubsection{Rémanence des formulations}

Les différentes formulations, bien qu'efficaces dans le piégeage de B. dorsalis particulièrement, ne présentent pas la même rémanence. Elle est très 
longue chez le méthyl eugénol comparativement à celle des formulations du basilic. Elle est estimée à 119,67 $\pm 40,5$ jours en moyenne au niveau du méthyl eugénol. Pour les formulations du basilic, la plus longue rémanence est observée avec la PB ( $28 \pm 18,18$ jours) et la plus faible avec le MB avec 5,33 $\pm 2,31$ jours (Figure 3 ). L'analyse de variance révèle une différence hautement significative entre la durée d'action des formulations.

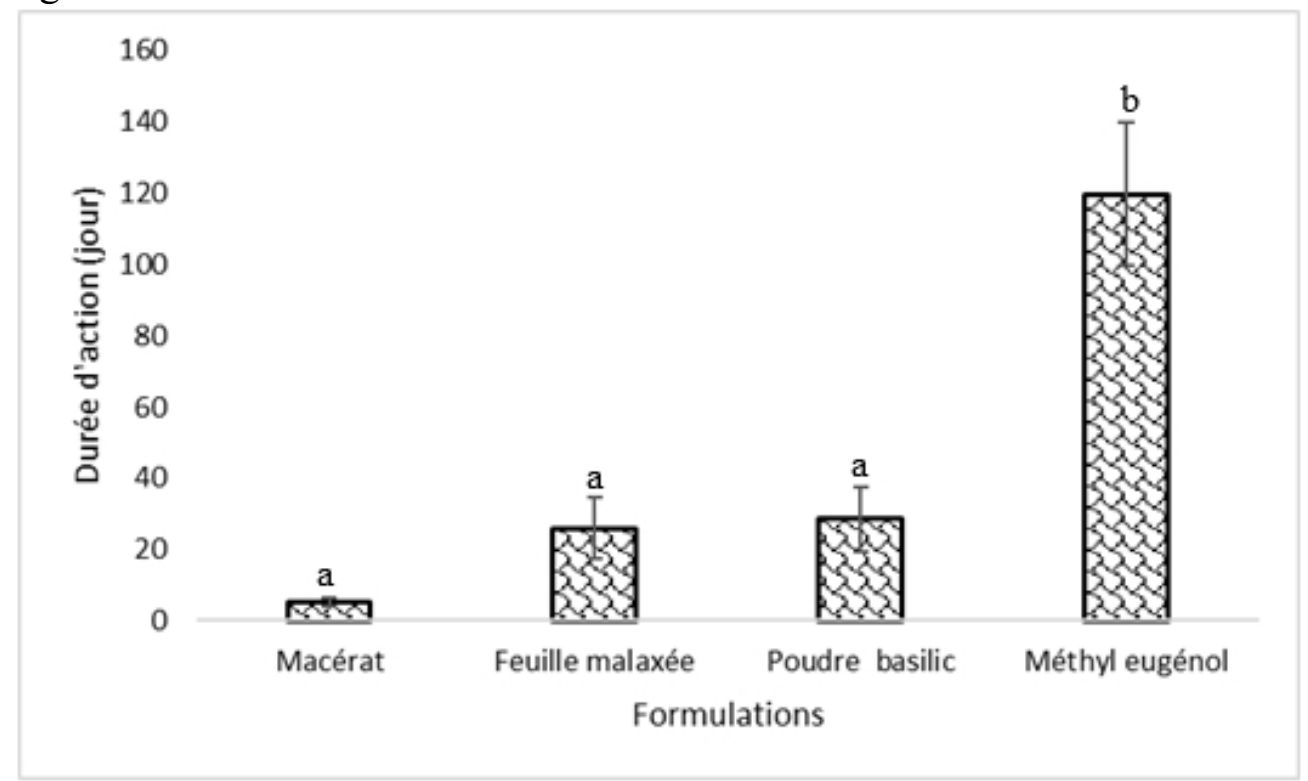

Figure 3 : Durée d'action des différentes formulations

ANOVA à $5 \% \quad \mathrm{dl}=3 \quad \mathrm{~F}=13,88 \quad \mathrm{P}=0,001$

Les moyennes affectées de la même lettre ne sont statistiquement significatives

\section{2.- Discussion}

Lors du piégeage avec les différentes formulations du basilic (Feuille Malaxée, Poudre de basilic et macérât de basilic), diverses espèces ont été capturées. Il s'agit de D. bivittatus, D. punctatifrons, D. langi, T. coffeae, C. anonae, C. punctata, C. bremii et la mouche invasive Bactrocera dorsalis. Ces espèces ont été, pour la plupart, identiques à celles capturées avec le méthyl eugénol. La mouche exotique invasive est dominante au niveau des pièges. La forte prévalence de $\mathrm{B}$. dorsalis observée par rapport aux autres espèces est également soulignée par Amevoin et al. (2009) lors de leurs travaux au Togo. Elle pourrait être due à une bonne adaptation aux conditions de la région (facteurs climatiques), à son caractère agressif vis-à-vis des autres espèces et à son statut de super compétiteur (forte aptitude à exploiter les plantes-hôtes). Cette mouche a été fortement attirée par le méthyl eugénol tout comme le basilic. N'Dépo (2010), Coulibaly (2015) ; N'Dépo et al. (2015) ont constaté une importante capture de $\mathrm{B}$. dorsalis à l'aide du méthyl eugénol en vergers de manguiers et en zone de forêt à Korhogo, dans la région nord de la Côte 
d'Ivoire. Yao (2018) signale une forte invasion de B. dorsalis dans les pièges appâtés au méthyl eugénol dans la localité de Daloa, au Centre Ouest du pays. En dehors du piégeage, l'abondance de cette même espèce au sein des fruits collectés, les mangues greffées en particulier, sur le marché local de Daloa, a été également souligné (Assi, 2018). En effet, le méthyl eugénol (4-allyl-1,2dimethoxybenzene-carboxylate) est un puissant attractif chimique des mâles des mouches de fruits, en particulier le genre Bactrocera et le sous genre Ceratitis (Pardalaspis) (White \& Elson-Harris, 1992 ; Minhibo et al., 2018). Cette substance chimique est contenue à des proportions variables dans certaines plantes Lamiaceae (famille de la menthe) à laquelle appartient Ocimum basilicum (Jacques \& Aké, 1987 ; Dewit, 1965 ; Anonymes, 2007a). L'importante capture de B. dorsalis par le basilic s'expliquerait par une forte diffusion du phénylpropanoïde (méthyl eugénol), comme l'un des principaux constituants de la plante (Anonymes, 2007b). En effet, cet appât naturel avec une proportion variable de méthyl eugénol en son sein, se comporte comme un attractif sexuel (méthyl eugénol), d'où l'attraction de B. dorsalis dans les pièges appâtés au basilic. En plus du phénylpropanoïde, on y rencontre également d'autres composés tels que l'huile essentielle (moins de 1\%), des composés aromatiques (le cineol, le linalol, le citral, le méthyl chavicol, l'eugénol, le cinnamate méthylique), des monoterpènes et sesquiterpènes dans la plante (Anonymes, 2007b). Ngom et al. (2014) relèvent que les monoterpènes oxygénés dont l'estragol $(27,85-38,78 \%)$, le linalol $(18,45-$ $18,87 \%)$ et le méthyl-eugénol (9,98-14,40\%) sont majoritaires dans l'huile essentielle d'Ocimum basilicum. Par ailleurs, la mouche exotique a pour fruithôte préféré la mangue et elle pullule dans les zones de production de mangues (N'Dépo et al., 2019). Ainsi, la forte population de B. dorsalis serait-elle liée à une proportion élevée du méthyl eugénol dans les pièges. A l'île de Mayotte, il a été signalé une importante attractivité des mouches de fruits sur le maïs, utilisé comme plante piège en bordure de parcelle de courgettes (Thomas, 2015). Le basilic pourrait être utilisé également comme plante piège contre les mouches de fruits en zone de culture agricole. Cet appât pourrait être utilisé à grande échelle afin de contribuer à la réduire les pertes de mangues.

\section{Conclusion}

Le basilic (Ocimum basilicum) attire fortement la mouche de la mangue Bactrocera dorsalis. Toutes les trois (3) formulations ont été efficaces pour la détection et le piégeage de B. dorsalis. La formulation " Poudre de basilic »donne la rémanence la plus longue (28,67 jour) et la celle du « Macérât » du basilic donne la capture hebdomadaire moyenne la plus importante (491 individus).

Le Macérât de basilic attire et permet de capturer B. dorsalis au même titre que le méthyl eugénol en verger de manguiers. 
Il ressort de cette étude que la Poudre de feuilles sèches de basilic et le Macérât de feuilles fraîches de basilic peuvent être recommandées auprès des producteurs de mangues dans la lutte contre B. dorsalis.

\section{References:}

1. Achiépo G. M. (2019). Evaluation des pièges locaux et fluctuation des populations des mouches des fruits (Diptera: Tephritidae) dans la région du Haut-Sassandra (Centre-Ouest, Côte d'Ivoire) : cas du site de l'Université Jean Lorougnon Guédé. Mémoire de Master, Université Jean Lorougnon Guédé, Daloa (Côte d'Ivoire), 35 p.

2. Amevoin K., Sanbena B. B., Nuto Y., Gomina M., De Meyer M., Glitho I. A. (2009). Les mouches des fruits (Diptera: Tephritidae) au Togo : inventaire, prévalence et dynamique des populations dans la zone urbaine de Lomé. International Journal of Biological and Chemical Sciences 3 (5), 912-920. Extrait de http://indexmedicus.afro.who.int

3. Anonymes (2007a). Basilic (Ocimum basilicum). Extrait de http://www-ang.kfunigraz.ac.at/, Consulté le 26 décembre 2007.

4. Anonymes (2007b). Basilic (Ocimum basilicum). Extrait de http://www-ang.kfunigraz.ac.at/, Consulté le 26 décembre 2007.

5. Assi E. (2018). Inventaire et incidence des mouches des fruits (Diptera : Tephritidae) sur les fruits vendus sur les marchés de Daloa, Centre Ouest de la Côte d'Ivoire. Mémoire de Master 2, l'Université Jean Lorougnon Guédé, Daloa (Côte d'Ivoire), 47 p.

6. (CIPV) (2016). Établissement de zones exemptes de mouches des fruits (Tephritidae). Normes Internationales pour les Mesures Phytosanitaires, NIMP 26, FAO, Italie (Rome) 64 p.

7. Coulibaly A. (2015). Evaluation des différents systèmes de piégeage des mouches des fruits. Mémoire de Master 2, Université Jean Lorougnon Guédé, Daloa (Côte d'Ivoire), 47 p.

8. 8 Daniel J. P., Serge Q., Alain R., Philippe R., Vayssières J. F. (2015). Les mouches des fruits (Diptera: Tephritidae) identifiées dans la partie sud d'Haïti, Entomologie Faunistique - Faunistic Entomology, (68), 193-200. Extrait de Cirad-Agritrop (https://agritrop.cirad.fr/578403/)

9. De Meyer M., Salah M and White I. M. (2007). Invasive Fruit Fly Pest in Africa: A diagnostic tool and information reference for the four Asian species of fruit fly (Diptera, Tephritidae) that have become accidentally established as pest in Africa, including the India Ocean Island. Lettre d'information, $\mathrm{N}^{\circ} 1,31 \mathrm{p}$.

10. Dewit H. (1965). Les plantes du monde : les plantes à fleurs. Tome II, Hachette, Paris, 339 p. 
11. Dramane K. D. (2019). Diversité des mouches de fruits (Diptera : Tephritidae) des zones de faibles productions fruitières (Daloa : Côte d'Ivoire). Mémoire de Master 2, l’Université Jean Lorougnon Guédé, Daloa (Côte d'Ivoire), $42 \mathrm{p}$.

12. Drew R. A. I., Tsuruta K. and White I. M. (2005). A new species of pest fruit fly (Diptera: Tephritidae: Dacinae) from Sri Lanka and Africa. African Entomology, 13 (1): 149-154.

13. Ekesi S., Nderitu P. W. and Chang L. C. (2007). Adaptation to and small-scale rearing of invasive fruit fly Bactrocera invadens (Diptera: Tephritidae) on artificial diet. Annual Entomological Society of America, $100 \quad$ (4): 562-567. doi: 10.1603/00138746(2007)100[562:ATASRO]2.0.CO;2

14. Ekesi S., Nderitu P. W. and Rwomushana I. (2006). Field infestation, life history and demographic parameters of the fruit fly Bactrocera invadens (Diptera: Tephritidae) in Africa. Bulletin of Entomological Research, (96), 379-386. doi : https://doi.org/10.1079/BER2006442

15. Nugnes F., Russo E., Viggiani G. and Umberto B. (2018). First Record of an Invasive Fruit Fly Belonging to Bactrocera dorsalis Complex (Diptera: Tephritidae) in Europe. Insects, 9 (182), 1-11. doi:10.3390/insects9040182

16. Hala N. F., Quilici S., Gnago A. J., N’Dépo O. R, Kouassi K. P., Allou K. R. (2006). Status of fruit flies (Diptera: Tephritidae) in Côted'Ivoire and implications for mango exports. Proceeding of $7^{\text {th }}$ International Symposium on Fruit Flies of Economic Importance, sept. 10- 15, 2006, Salvadore, Bahia, Brazil. p 233-239.

17. Ivan R., Ekesi S., Gordon I. and Callistus k. P. O. O. (2008). Host plants and Host plant preference studies for Bactrocera invadens (Diptera: Tephritidae) in Kenya, a new invasive fruit fly species in Africa. Annual Entomological Society of America, 101 (2); 331-340. doi: 10.1603/0013-8746(2008)101[331:HPAHPP]2.0.CO;2

18. Jacques C. and Aké A. L. (1987). Plantes médicinales tropicales et ivoiriennes. Editions imprintex Breda (Hollande), diffusion exclusive en Côte-d'Ivoire, DARENI Editions les DEUX PLATEAUX, Abidjan, Tome I, $212 \mathrm{p}$.

19. Lux S. A., Copeland R. S., White I. M., Manrank A., Han A., Billah M. K. (2003). A new invadens fruit fly species from the Bactrocera dorsalis (Hendel) group detected in East Africa. In: A new species of pest fruit fly (Diptera: Tephritidae: Dacinae) from Sri Lanka and Africa. Drew R. A. I., Tsuruta K., White I. M. (eds). African Entomology, 13 (1), 149-154. doi: 10.1017/S174275840001242X

20. Minhibo M. Y., N’Dépo O. R, Hala N. F, Koua H, Tuo Y, N'Goran A., Coulibaly A., Doumbia S., et al., (2018). Assessment of Fruit Fly 
Trapping System in Mango Orchards in Northern Côte d'Ivoire. Journal of Agricultural Science and Technology A (8), 18-27. doi: $10.17265 / 2161-6256 / 2018.01 .003$

21. N'Dépo O. R. (2006). Inventaire des mouches des fruits (Diptera: Tephritidae) dans les stations expérimentales d'Azaguié et de Yamoussoukro (Côte-d'Ivoire). Mémoire D.E.A d'Entomologie Générale, Université Felix Houphouët Boigny; Abidjan (Côte d'Ivoire), $59 \mathrm{p}$.

22. N'Dépo O. R. (2010). Biologie et écologie de Bactrocera invadens Drew, Tsuruta et White, 2005 (Diptera : Tephritidae), principal déprédateur des vergers fruitiers en Côte-d'Ivoire : possibilité de lutte chimique raisonnée contre les mouches des fruits. Thèse de Doctorat, Université Felix Houphouët Boigny ; Abidjan (Côte d'Ivoire), p. 177.

23. N'Dépo O. R., Hala N. F., Allou K., Aboua L. R., Kouassi K. P., Vayssières J. F., et al., (2009). Abondance des mouches des fruits dans les zones de production fruitière de Côte-d'Ivoire : dynamique des populations de Bactrocera invadens (Diptera : Tephritidae). Fruits, 64 (5), 313-324. doi: 10.1051/fruits/2009028

24. N'Dépo O. R., Hala N. F., Gnago A., Allou K., Kouassi K. P., Vayssières J. F., et al., (2010). Inventaire des mouches des fruits de trois régions Agro-écologiques et plantes-hôtes associées à l'espèce nouvelle Bactrocera (Bactrocera) invadens Drew et al. (Diptera : Tephritidae) en Côte-d'Ivoire. European Journal of Scientific Research, $46 \quad$ (1), 63-72. Extrait de http://www.eurojournals.com/ejsr.htm

25. N'Dépo O. R., Hala N, N'Da A. A., Coulibaly F., Kouassi K. P., Vayssieres J. F., et al., (2015). Effective chemical control of fruit flies (Diptera : Tephritidae) pests in mango orchards in northern Côted'Ivoire. Int. J. Biol. Chem. Sci., 9 (3), 1299-1307. Extrait de doi: http://dx.doi.org/10.4314/ijbcs.v9i3.15

26. N'Dépo O. R., Minhiba M. Y., N'Goran A., Hala N. F., Coulibaly A., Soro S., et al., (2019). Host plants associated with Tephritidae in Côte d'Ivoire and discovery of a new fruit fly species : Dacus longistylus. Journal of Entomology and Zoology Studies, 7 (3), 1301-1308. Extrait de http:// www.entomoljournal.com

27. Ngom S., Diop M., Mbengué M., Faye F., Kornprobst J. M., Samb A. (2014). Composition chimique et propriétés antibactériennes des huiles essentielles d'Ocimum basilicum et d'Hyptis suaveolens (L.) Poit récoltés dans la région de Dakar au Sénégal. Afrique SCIENCE 10 (4), 109-117. Extrait de http://www.afriquescience.info 
28. Ouattara M. (1998). Fluctuation des populations des mouches des fruits (Diptera: Tephritidae) dans la région de Korhogo. Mémoire INP/ESA, Côte d'Ivoire, $46 \mathrm{p}$.

29. 29 Ouedraogo S. N., Vayssières J. F., Dabiré A. R., Lefèvre C. R. (2011). Biodiversité des mouches des fruits (Diptera : Tephritidae) en vergers de manguiers de l'ouest du Burkina Faso : structure et comparaison des communautés de différents sites. Fruits, (66), 393404. doi: $10.1051 /$ fruits/2011054

30. Tinkeu L. N., Ladang D., Vayssières, J-F., Lyannaz J. P. (2010). Diversité des espèces de mouches des fruits (Diptera : Tephritidae) dans un verger mixte dans la localité de Malang (Ngaoundéré, Cameroun). International Journal of Biological and Chemical Sciences, 4 (5), 1425-1434. Extrait de http://dx.doi.org/10.4314/ijbcs.v4i5.65530

31. Thomas C. (2015). Les mouches des Cucurbitacées. Rapport de synthèse, 23p.

32. Vayssières J. F., Georg G., Orphée L., Dossa P., Akponon C. (2005). A new Bactrocera species in Benin among fruit fly (Diptera: Tephritidae) species. Fruits, 60 (6), 371-377. doi: 10.1051/fruits:2005042

33. Vayssières J. F., Sinzogan A., Korie S., and Odjo A. T. (2009). Effectiveness of spinosad bait sprays (GF-120) in controlling mangoinfesting fruit flies (Diptera: Tephritidae) in Benin. Journal of Economic Entomology, 102 (2), 515-521. doi: 10.1603/029.102.0208

34. White I. M. and Elson-Harris M. M. (1992). Fruit flies of economic significance, ACIAR, Wallingford, Oxon, UK , CABI, $601 \mathrm{p}$.

35. Yao L. I. D. (2018). Diversité des mouches des fruits (Diptera: Tephritidae) et leurs plantes-Hôtes au Centre Ouest de la Côte d'Ivoire : cas du site de l'Université Jean Lorougnon Guédé (Daloa). Mémoire de Master 2, Université Jean Lorougnon Guédé, Daloa (Côte d'Ivoire) $37 \mathrm{p}$. 